\title{
Declarations: management of a pulmonary arteriovenous fistulae by uniportal video- assisted thoracoscopic surgery: a case report
}

\author{
R. Li', Y. Zhou' ${ }^{1}$ S. Kang ${ }^{2}$, F. Kong ${ }^{1}$, L. Guan ${ }^{1}$, Y. Zhao ${ }^{1}$ and X. Yin ${ }^{1 *}$ (i)
}

\begin{abstract}
Background: A pulmonary arteriovenous fistula (PAVF) is arare condition that is associated with pulmonary arteriovenous malformation(PAVM). Few reports have described managing PAVMs using uniportalvideo-assisted thoracoscopic surgery (VATS).

Case presentation: A 13-year-old child with PAVF in the left inferior pulmonary artery was treated by uniportal VATS with left lower lobectomy. After surgery, hemoptysis did not recur and there were no postoperative complications. Six months after the operation, postoperative review of computerized tomography showed no recrudescence of PAVF.

Conclusions: PAVF is a rare case that should be diagnosed and treated early. 3D-computerized tomography (CT) reconstruction is useful for diagnosis and preoperative assessment. The case shows that PAVF can be managed with uniportal VATS.
\end{abstract}

Keywords: Pulmonary arteriovenous fistula, 3D-CT reconstruction, Uniportal VATS, Case report

\section{Background}

A pulmonary arteriovenous fistula (PAVF) is a rare condition, first described by Churton in 1897 [1], that is associated with pulmonary arteriovenous malformation (PAVM), which allows abnormal direct communication between the pulmonary arteries and pulmonary veins [2]. The most common cause of PAVM is hereditary hemorrhagic telangiectasia, also known as OslerWeber-Rendu syndrome [3]. Patients without obvious symptoms account for approximately $57 \%$ of PAVF cases in the early stage of the disease. However, PAVF can also exhibit diverse symptoms, including repeated hemoptysis, nosebleeds, difficulty catching breath, an increase in hemoglobin levels, and hemoptysis, which can lead to sudden fatal rupture of the veins $[4,5]$. Patients with

\footnotetext{
*Correspondence: xiaochuanyin1@126.com

1 Department of Thoracic Surgery, The First Affiliated Hospital of Kunming

Medical University, Kunming 650032, Yunnan, China

Full list of author information is available at the end of the article
}

these symptoms are treated with interventional therapy [6] or surgical procedures including lung wedge resection, lobectomy, and pneumonectomy $[7,8]$. The following is a case report of a 13-year-old patient with PAVF in the left inferior pulmonary. The patient, who exhibited hemoptysis and dyspnea, was diagnosed by preoperative 3D-computed tomography (3D-CT) reconstruction and treated by uniportal video-assisted thoracoscopic surgery (VATS).

\section{Case presentation}

A 13-year-old child developed symptoms of mild hemoptysis and dyspnea that persisted for more than two months and did not respond to oral medication. Physical examination showed only a mild cyanosis of lips, with no other obvious findings. The patient had no relevant previous medical history and there was no relevant family history. Chest radiography indicated that a mass was located in the left inferior pulmonary. Non-enhanced chest computerized tomography (CT) and subsequent computerized tomographic angiography (CTA) confirmed 
a large $3.8 \times 3.2 \mathrm{~cm}$ lesion in the left lower lung lobe (Fig. 1a, b). A 3D-CT reconstruction [9] made by Mimics Medical 21.0 confirmed what appeared to be a PAVM or PAVF before surgery, which was directly connected between $\mathrm{A}^{7-8}$ of left lower pulmonary arteries and $\mathrm{V}^{6-8}$ of left lower pulmonary veins not feeder vessels (Fig. 2a, b). Bronchoscopy indicated that normal tracheobronchial anatomy was affected by the endobronchial lesion and by airway compression. A routine blood examination showed that hemoglobin was $113.0 \mathrm{~g}$ (normal range: $120-172$ ), hematocrit $47 \%$ (normal range: $36-50 \%$ ), arterial blood gas $\mathrm{PaO}_{2} 59 \mathrm{mmHg}$ (normal range: 83-108) and $\mathrm{SO}_{2} 93 \%$ (normal range: $95-98 \%$ ).

Uniportal VATS was carried out through a $3 \mathrm{~cm}$ incision in the fifth intercostal anterior-axillary space. A dark blue mass on the left interlobar fissure was found via thoracoscopy and was concluded to be a PAVM (Fig. 3). The lobectomy began by releasing pleuropulmonary adhesions and the inferior pulmonary ligament and dissecting the aortopulmonary to expose the left pulmonary arterial trunk (LPAT), which was controlled proximally by using a vascular tourniquet. The major fissure was then divided to identify the left inferior pulmonary artery. The left inferior pulmonary vein (LIPV) and the left superior pulmonary vein (LSPV) were exposed after dissecting the hilum of left pulmonary arteries. The large PAVM was directly connected between $\mathrm{A}^{7-8}$ of left lower pulmonary arteries and $\mathrm{V}^{6-8}$
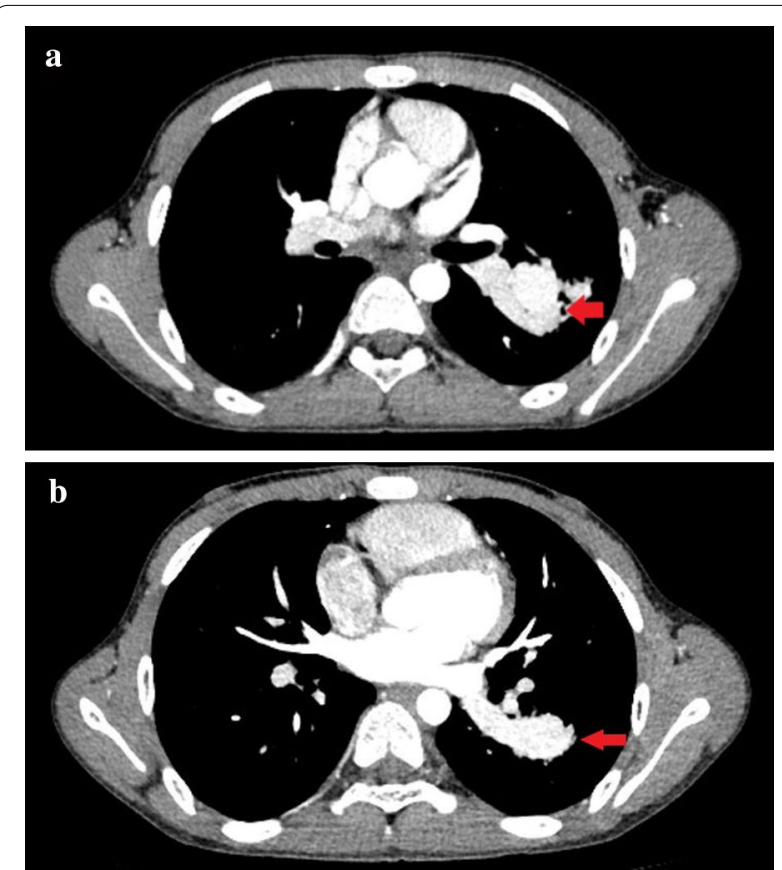

Fig. 1 a, b CTA scan showed a large $3.8 \times 3.2 \mathrm{~cm}$ lesion in the left lower lobe. (Mediastinal window)
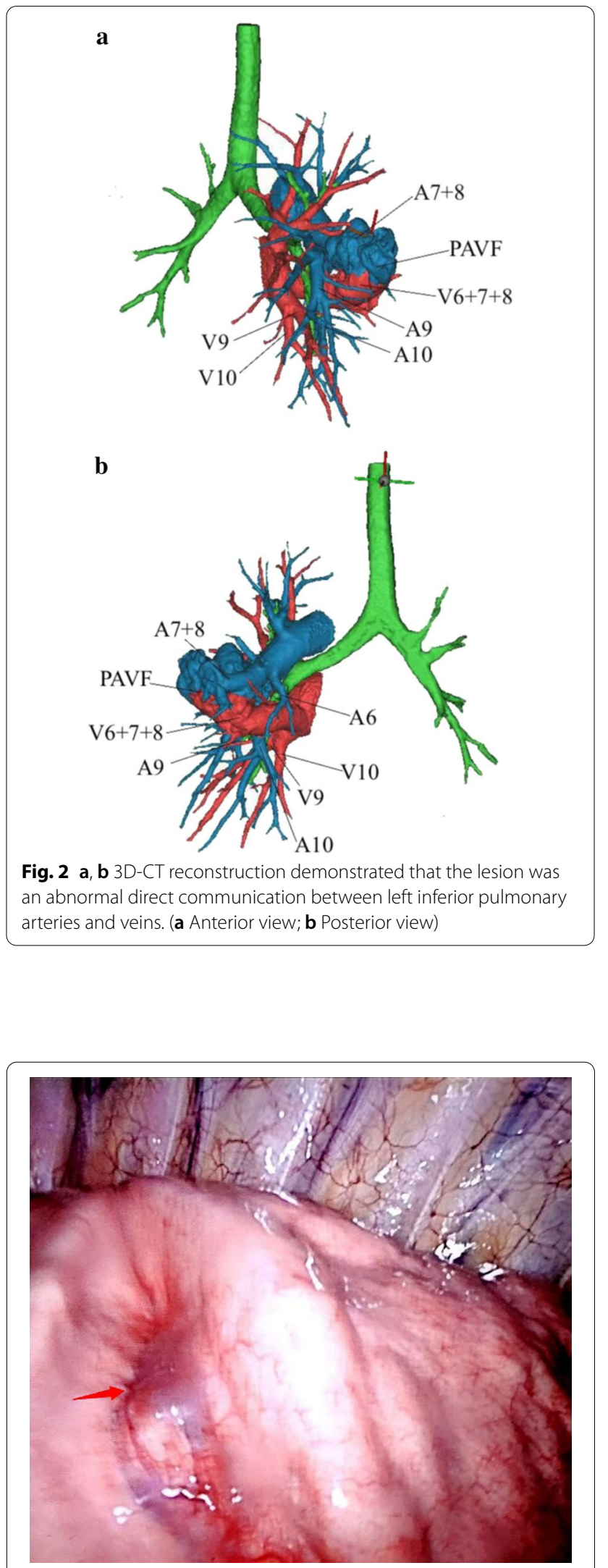

Fig. 3 A dark blue mass was found on the left interlober fissure 
of left lower pulmonary veins, and this was consistent with the results of preoperative $3 \mathrm{D}-\mathrm{CT}$ reconstruction. The left inferior pulmonary artery and vein were ligated using staplers (Fig. 4a, b). The bronchus of the left lower lobe was then clamped by using staples and removed during the operation. A pathological diagnosis of PAVF was confirmed (Fig. 5).

Hemoptysis and postoperative complications did not occur after surgery. The tube was removed after reviewing the chest X-ray film for pneumothorax and abnormal pleural effusion. The patient was discharged from hospital five days after surgical resection. Six months after the operation, postoperative review of computed tomography showed no recrudescence of PAVF (Fig. 6).

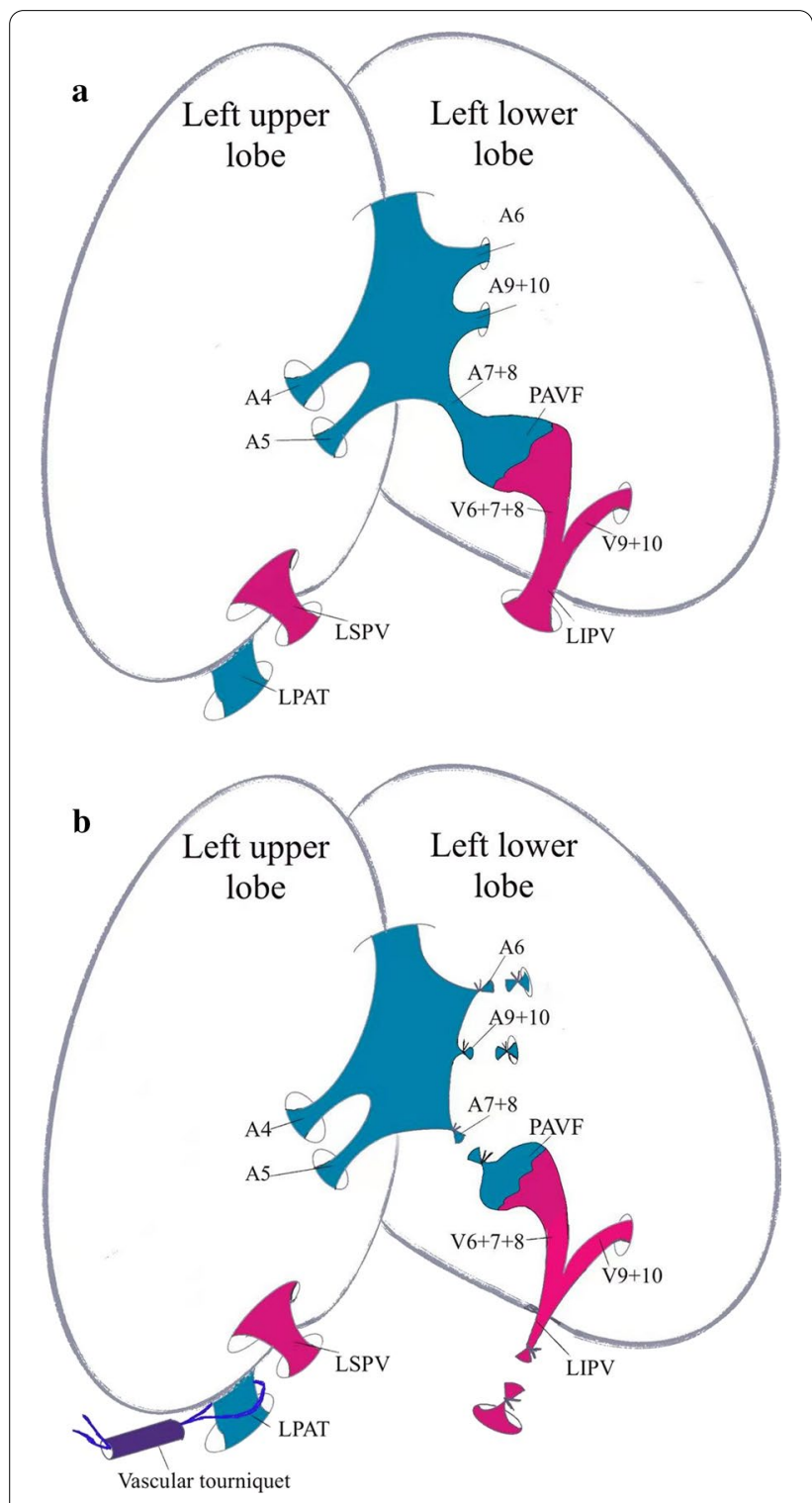

Fig. 4 Sketch profile of the pulmonary arteriovenous fistula

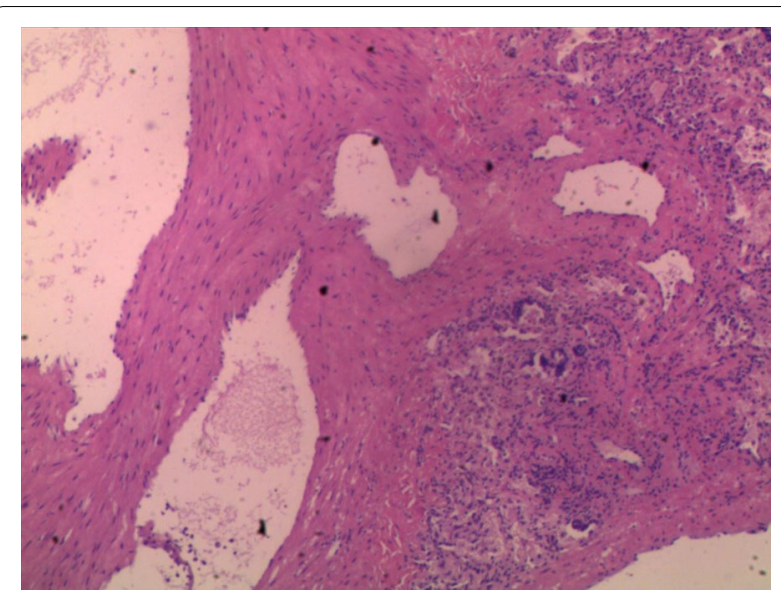

Fig. 5 A pathological diagnosis of PAVF

\section{Discussion and conclusions}

The condition of PAVF occurs mainly due to defects in pulmonary capillary development. Abnormal communications between pulmonary arteries and veins form a right-to-left shunt causing blood flow without oxygenation and an increase in red cells. Complications may be caused by PAVMs due to direct intercommunication between pulmonary arteries and pulmonary veins, including exertional dyspnea, swirl, cyanopathy, polycythemia, bacterial infections, and brain abscesses. It can appear as a mass on routine chest radiography and abnormal direct communication between pulmonary arteries and pulmonary veins on CT or 3D-CT.

Appropriate therapies for PAVF are interventional therapy or surgical treatment. As in this case, a patient with serious PAVM and hemoptysis should be treated to prevent possible severe dyspnea due to life-threatening hemorrhage and hypoxemia caused by PAVM. In this case, because the diameter of the PAVF was limited to a lobe and was larger than $2 \mathrm{~cm}$, with a.

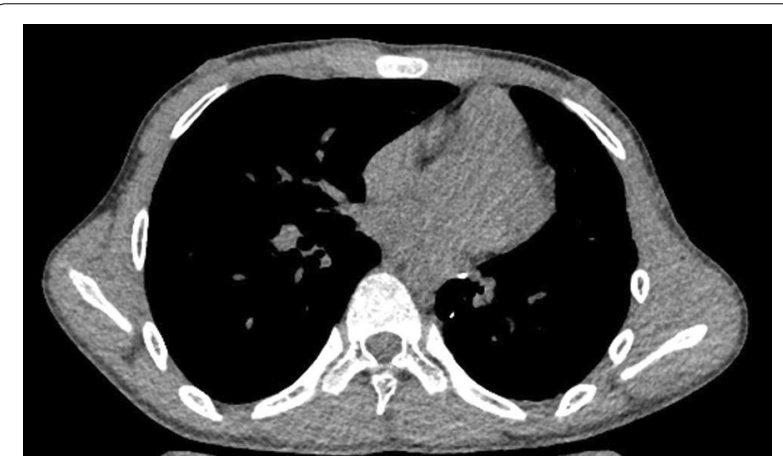

Fig. 6 Postoperative review of computed tomography showed no recrudescence of PAVF. (Mediastinal window) 
feeding artery over $3 \mathrm{~mm}$ in diameter, it was decided that interventional therapy would not solve the problem and that a surgical procedure should be chosen if the operative risk could be controlled. Because the PAVF of the patient was located deeply, it was hard to remove using transcatheter embolotherapy and lung wedge resection. Therefore, left lower lobectomy was selected.

Three points were analyzed to reduce the thoracoscopic surgical risk during this operation. The 3D-CT reconstruction was used to confirm the structure of the PAVM before the operation. Preoperative simulation based on $3 \mathrm{D}-\mathrm{CT}$ reconstruction is useful for accurate diagnosis, and in this case, 3D-CT reconstruction revealed a huge malformed hemangioma that was directly connected between the trunk of left lower pulmonary arteries and the left lower pulmonary veins not feeder vessels, which was coincident with the intraoperative situation. The use of 3D-CT reconstruction also showed the anatomical variation of the blood vessels, so life-threatening hemorrhage could be avoided during surgery. Risk analysis through 3D-CT reconstruction indicated that the left pulmonary trunk should be controlled proximally by using a vascular tourniquet to avoid uncontrolled arterial bleeding. This control of the left pulmonary trunk allowed the distally involved pulmonary parenchyma to be safely resected. More importantly, precise patient positioning for thoracoscopic pulmonary lobectomy allowed a suitable surgical plan for the operating surgeon and reduced the risks involved in thoracoscopic surgery. Few cases of PAVF have been published, so no outcome data were available for this surgical procedure. Uniportal VATS was performed for the PAVF in this case, because it was a secure and feasible technique in thoracic surgery and provided a better surgical field. To reduce surgical trauma, there was only one surgical hole. As such, there were fewer complications, less pain, and, consequently, a shorter postoperative hospital stay [10].

In general, PAVF is a rare case which should be diagnosed and treated early. 3D-CT reconstruction may be useful for diagnosis and preoperative assessment. The case could provide the experience and feasibility for PAVF managed with uniportal video-assisted thoracoscopy.

\footnotetext{
Abbreviations

PAVF: Pulmonary arteriovenous fistulae; PAVM: Pulmonary arteriovenous malformation; 3D-CT:Three dimensional-computed tomography; VATS: Videoassisted thoracoscopic surgery; CT: Computed tomography; CTA: Computed tomograhy angiograhy; LPAT: Left pulmonary arterial trunk; LIPV: Left inferior pulmonary vein; LSPV: Left superior pulmonary vein.
}

\section{Acknowledgements}

We would like to thank X Yin and Y Zhao, at The First Affiliated Hospital of Kunming Medical University, for study design and critical review. We thank
International Science Editing (http://www.internationalscienceediting.com) for editing this manuscript.

\section{Authors' contributions}

$\mathrm{RL}$ is the first author of the manuscript. SK made the 3D-CT reconstruction. $Y Z$, FK and $L G$ treated the patient after surgery. $X Y$ and $Y Z$ performed the operation, and substantially contributed to the drafting and revision of the manuscript. $X Y$ is the corresponding author. All authors read and approved the final manuscript.

\section{Funding}

None.

Availability of data and materials

The data and materials sets support the conclusions of this article.

Ethics approval and consent to participate

Not applicable.

\section{Consent for publication}

Written informed consent for the publication of these case reports and accompanying images was obtained from the guardians of our patients.

\section{Competing interests}

All authors declare that they have no competing interests.

\section{Author details}

${ }^{1}$ Department of Thoracic Surgery, The First Affiliated Hospital of Kunming Medical University, Kunming 650032, Yunnan, China. ${ }^{2}$ Department of Imaging, The First Affiliated Hospital of Kunming Medical University, Kunming 650032 Yunnan, China.

Received: 30 September 2020 Revised: 11 February 2021 Accepted: 15 February 2021

Published online: 23 February 2021

\section{References}

1. Churton T. Leeds and west-riding medico-chirurgical society: multiple aneurysms of pulmonary artery. Brit Med J. 1897;1:1223.

2. Shovlin CL. Pulmonary arteriovenous malformations. Am J Respir Crit Care Med. 2014;190:1217-28.

3. Holzer RJ, Cua CL. Pulmonary arteriovenous malformations and risk of stroke. Cardiol Clin. 2016;34:241-6.

4. Gh O, Jb S, Aw S, et al. Paradoxical cerebrovascular embolism associated with pulmonary artefiovenousfistula: contrast transoesophageal echocardiographic diagnosis. Eur J Echocardiogr. 2001;2(3):207-11.

5. Liao Y, Chen KH, Huang GY, Song W. Pulmonary arteriovenous malformations presenting as refractory heart failure. J Thorac Dis. 2014;6(9):E169-72.

6. Contegiacomo A, Ciello AD, Rella R, et al. Pulmonary arteriovenous malformations: what the interventional radiologist needs to know. La radiologia medica. 2019:124:10.

7. Yufei Wang K, Wang C, Guo Z, Guo. Successful treatment of multiple pulmonary arteriovenous fistulae with thoracoscopy. Thorac Cancer. 2018;9(8):1082-6.

8. Teng P, Li W, Ni Y. Surgical lobectomy of pulmonary arteriovenous malformations in a patient with presentations regarded as sequela of tuberculosis: a case report. J Cardiothorac Surg. 2020. https://doi.org/10.1186/s13019-02001319-4.

9. Fan C, Cheng J, Wu S, et al. Pulmonary arteriovenous malformation detected by three-dimensional computed tomographic angiography. Heart Lung Circ. 2017;26(8):e59-61.

10. Gonzalez-Rivas. Diego. Unisurgeon' uniportal video-assisted thoracoscopic surgery lobectomy. J Vis Surg. 2017;3:163.

\section{Publisher's note}

Springer Nature remains neutral with regard to jurisdictional claims in published maps and institutional affiliations. 